\title{
Production of oleanane-type sapogenin in transgenic rice via expression of $\beta$-amyrin synthase gene from Panax japonicus C. A. Mey
}

\author{
Zhiwei Huang ${ }^{1,2}$, Juncheng Lin², Zuxin Cheng ${ }^{2}$, Ming X ${ }^{2}$, Mingshu Guo ${ }^{2}$, Xinying Huang ${ }^{2}$, Zhijian Yang ${ }^{2}$ \\ and Jingui Zheng ${ }^{2^{*}}$
}

\begin{abstract}
Background: Panax japonicus C. A. Mey. is a rare traditional Chinese herbal medicine that uses ginsenosides as its main active ingredient. Rice does not produce ginsenosides because it lacks a key rate-limiting enzyme ( $\beta$-amyrin synthase, $\beta A S)$; however, it produces a secondary metabolite, 2,3-oxidosqualene, which is a precursor for ginsenoside biosynthesis.

Results: In the present study, the $P$. japonicus $\beta A S$ gene was transformed into the rice cultivar Taijing $9^{\prime}$ using an Agrobacterium-mediated approach, resulting in 68 rice transgenic plants of the $T_{0}$ generation. Transfer-DNA (T-DNA) insertion sites in homozygous lines of the $T_{2}$ generation were determined by using high-efficiency thermal asymmetric interlaced PCR (hiTAlL-PCR) and were found to vary among the tested lines. Approximately 1-2 copies of the $\beta A S$ gene were detected in transgenic rice plants. Real-time PCR and Western blotting analyses showed that the transformed $\beta A S$ gene could be overexpressed and $\beta$-amyrin synthase could be expressed in rice. HPLC analysis showed that the concentration of oleanane-type sapogenin oleanolic acid in transgenic rice was 8.3-11.5 mg/100 g dw.
\end{abstract}

Conclusions: The current study is the first report on the transformation of $P$. japonicus $\beta A S$ gene into rice. We have successfully produced a new rice germplasm, "ginseng rice", which produces oleanane-type sapogenin.

Keywords: Ginsenosides, Sapogenin, $\beta$-amyrin synthase gene, Rice, Genetic transformation

\section{Background}

Rice is one of the world's major food crops. It is the staple food for nearly half of the world's population [1]. Traditional breeding techniques, especially hybrid rice technology, have contributed to the improvement of rice yield and quality. However, there are compounds that rice cannot synthesize or can only produce at very low levels, and traditional breeding techniques are apparently incapable of resolving these problems. Transgenic technology provides an efficient means of improving rice quality at the genetic level [2-4] based on the principle that creating a new rice germplasm that is capable of producing exogenous active ingredients through genetic engineering could improve the nutritional quality of rice. For example, Ye et al. [5] and Paine et al. [6] produced

\footnotetext{
* Correspondence: zjg231@126.com

${ }^{2}$ Agricultural Product Quality Institute, Fujian Agriculture and Forestry University, 15 Shangxiadian Road, CangShan District, Fuzhou 350002, Fujian, China

Full list of author information is available at the end of the article
}

the first and second generations of "golden rice", respectively, mainly by expressing exogenous key genes in the $\beta$-carotene biosynthesis pathway in the endosperm. The reported concentration of $\beta$-carotene was $1.6 \mu \mathrm{g} / \mathrm{g}$ and $37 \mu \mathrm{g} / \mathrm{g}$ in these two transgenic rice strains, respectively. Lee et al. [7] transformed the sesame $2 S$ albumin gene into rice and produced "sesame nutrition rice", and the methionine content of the seeds reached $0.40 \%$ in the $\mathrm{T}_{2}$ generation rice lines, which represented a $38 \%$ increase relative to that of the control rice lines.

$P$. japonicus is a rare traditional Chinese herbal medicine that uses ginseng saponin as its main active ingredient. Its health-protective effects include improving immunity, preventing tumors, and facilitating adaptation $[8,9]$. The total saponin content in the roots of $P$. japonicus can reach $15 \%$, which is 2 - to 7 -fold higher than that of $P$. ginseng and 3-fold higher than that of $P$. quinquefolius $[8,9]$. Ginsenosides are triterpenoid saponins of plant secondary metabolites, i.e. the product of 


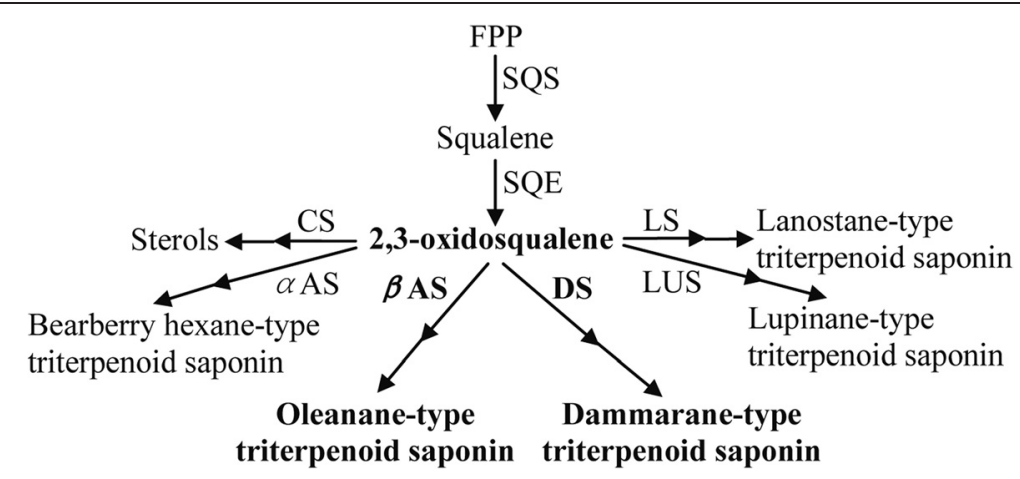

Fig. 1 Biosynthetic pathway of triterpenoid saponin and sterols in plants [10-12]. Intermediates: FPP, farnesyl pyrophosphate; SQS, squalene synthase; SQE, squalene epoxidase; CS, cycloartenol synthase; LS, lanosterol synthase; aAS, a-amyrin synthase; LUS, lupeol synthase; DS, dammarenediol-II synthase; $\beta A S, \beta$-amyrin synthase

triterpenoid saponins biosynthesis branch in the isoprenoid pathway (Fig. 1) [10-12].

Triterpenoid saponins are formed by different cyclization reactions of squalene. The synthesis of squalene is the branch point of the central isoprenoid pathway. It occurs at the triterpenoid saponin biosynthetic branch $[11,13,14]$. 2, 3-oxidosqualene cyclases (OSCs), the rate-limiting enzymes in triterpenoid saponins and sterols biosynthesis branches, catalyze 2,3-oxidosqualene, which results in the formation of the triterpenoid skeleton, cycloartenol, and other compounds. With further modifications, these compounds can form a variety of triterpenoid saponins, phytosterols, and other macromolecules.

The type and catalytic function of OSCs differ between ginseng plants and rice. The OSCs of ginseng plants are mainly $\beta$-amyrin synthase ( $\beta A S)$ and dammarenediol-II synthase (DS). These catalyze 2,3-oxidosqualene to produce oleanane-type and dammarane-type substances, namely, $\beta$-amyrin and dammarenediol-II, respectively. Rice OSC is a cycloartenol synthase (CS) that catalyzes 2,3-oxidosqualene to produce cycloartenol [6, 11, 14]. Zhao et al. [15] studied the expression of ginseng $\beta A S$ and the regulation of ginsenosides biosynthesis by using antisense RNA technology. When antisense $\beta A S$ was introduced into the hairy roots of ginseng, the transcription levels of the $\beta A S$ gene significantly decreased compared to those of non-transgenic controls. In addition, $\beta$ AS activity also decreased, and the concentration of oleanane-type ginsenosides Ro decreased by as much as $40 \%$. DS activity increased in these $\beta A S$ antisense lines, and the concentration of dammarane-type ginsenosides increased by up to $30 \%$. These results proved that regulation of ginsenosides synthesis could be achieved by altering the gene expression pattern of $\beta A S$ by using genetic engineering techniques.

The $\beta A S$ gene has been cloned from Panax ginseng (GenBank Acc. Nos.: AB014057.1, AB009030.1) [10], Aralia elata (GenBank Acc. No.: HM219225.1), Betula platyphylla (GenBank Acc. No.: AB055512.1), Malus domestica (GenBank Acc. No.: FJ032007.1), Pisum sativum (GenBank Acc. No.: AB034802.1) [10], and Centella asiatica (GenBank Acc. No.: AY520818.1). The $\beta A S$ gene has not been cloned from rice. However, rice OSC is a CS, which catalyzes 2,3-oxidosqualene to produce the sterol substrate, cycloartenol [10, 16-18]. This indicates that although 2,3-oxidosqualene, the precursor of rice sterols synthesis, is also the precursor for ginsenosides synthesis $[11,13]$, rice cannot synthesize ginsenosides because rice lacks $\beta$ AS and DS, which are the rate-limiting enzymes in the synthesis of ginsenosides.

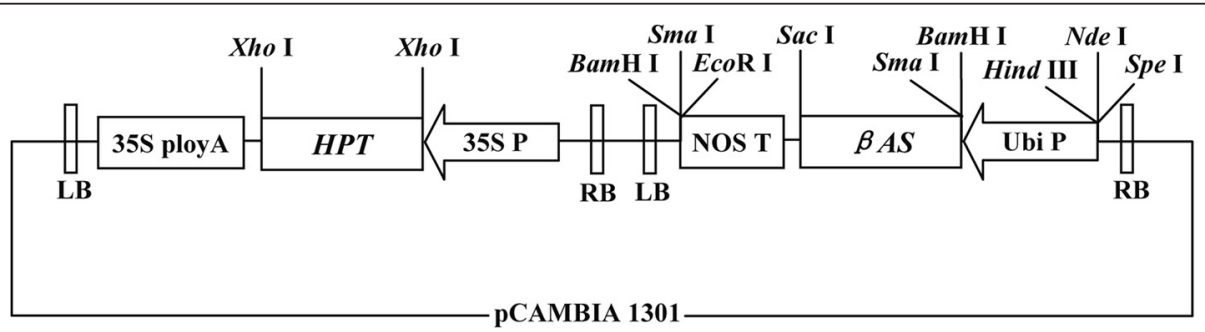

Fig. 2 T-DNA region of the plant expression vector PCD-AS-hpt. Intermediates: 35 S polyA, terminator of CaMV 35S gene; HPT, hygromycin phosphotransferase gene; $35 \mathrm{~S}$ P, $35 \mathrm{~S}$ promoter; NOS T, terminator of nopaline synthase gene; Ubi P, Ubiquitin promoter; LB, left border; $\mathrm{RB}$, right border 


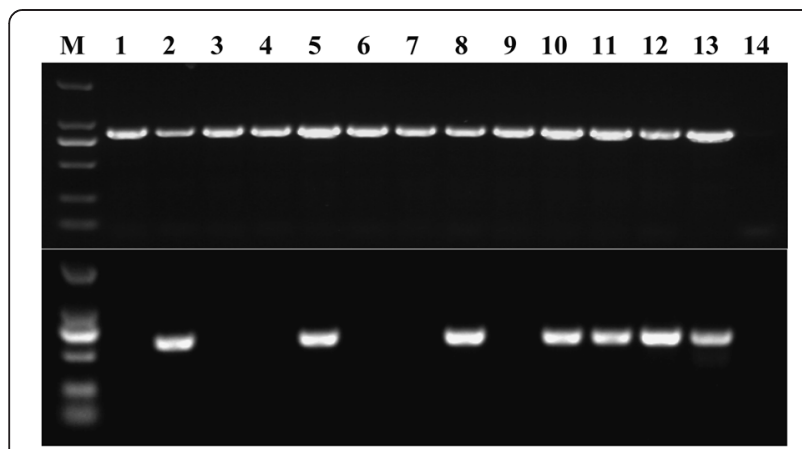

Fig. 3 PCR analysis of HPT (845 bp) and $\beta A S$ (584 bp) in transgenic rice plants. M, DNA Marker DL 2000. (1-12) Transgenic rice plants. (13), positive control (vector carrying the $\beta A S$ gene). (14) Negative control (rice cultivar 'Taijing 9')

In summary, genetic engineering of the secondary metabolic pathways allows rice to synthesize saponin. However, no report on the use of genetic engineering on rice to synthesize saponin currently exists. Previously, our research group isolated the full-length cDNA of $\beta A S$ (GenBank Acc. No.: KP658156) from the roots of $P$. japonicus, cloned this into the $\mathrm{pMD}^{\circ}$ 18-T Simple Vector (Takara, D103A), and named the construct as pMD-AS.

To create a new "ginseng rice" germplasm and to provide germplasm resources for further improvement of rice quality, the present study transformed the P. japonicus $\beta A S$ gene into rice to synthesize oleanane-type sapogenin.

\section{Methods}

\section{Experimental materials}

Rice cultivar 'Taijing 9', Escherichia coli (E. coli) strain DH5 $\alpha$, Agrobacterium tumefaciens (A. tumefaciens) strain LBA4404, plasmids pMD-AS, pMD-Gt1-AmA1, pBlue-Ubi, and pCD-AMA1-hpt were maintained by the Agricultural Product Quality Institute of Fujian Agriculture and Forestry University.

\section{Construction of a binary plant expression vector carrying the $\beta A S$ gene}

The construction of a binary plant expression vector harboring the $\beta A S$ gene was as described elsewhere [19]. Briefly, double digestion of plasmids pMD-AS by SmaI and $S a c$ I was conducted to isolate a DNA fragment that included the $\beta A S$ gene. The fragment was then recovered and ligated to SmaI and SacI double-digested pMD-Gt1-AmA1 vector to form pMD-Gt1-AS. The pBlue-Ubi plasmid was double-digested with HindIII and BamHI to release the Ubi promoter fragment. This fragment was then recovered and ligated to HindIII and BamHI double-digested pMD-Gt1-AS vector to form

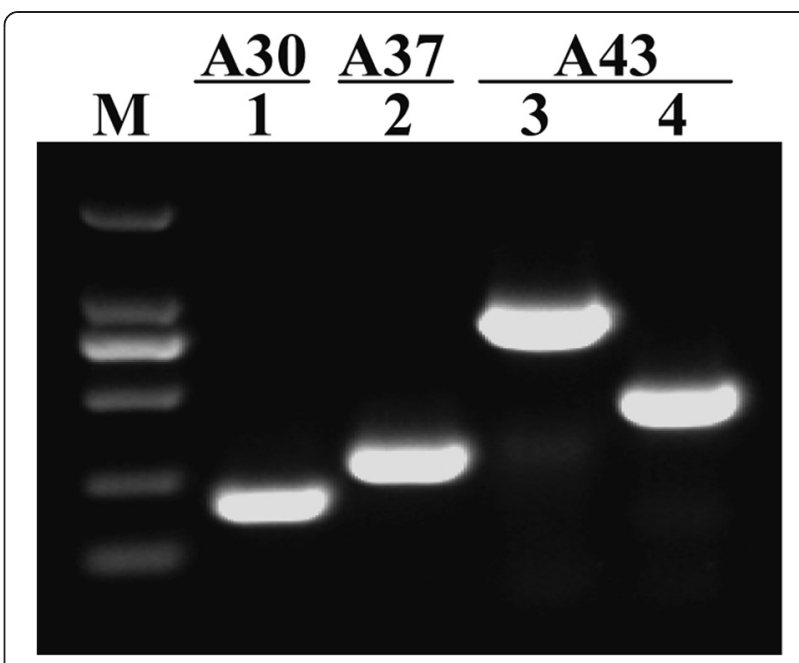

Fig. 4 PCR analysis of T-DNA flanking sequences from transgenic rice plants. M, DNA Marker DL 2000. (1-4), PCR products of the T-DNA flanking sequences

plasmid pMD-Ubi-AS. The pMD-Ubi-AS plasmid was double-digested with HindIII and SacI to release the Ubi- $\beta A S$ fragment. This fragment was then recovered and ligated to HindIII and SacI double-digested plasmid pCD-AMA1-hpt to form binary plant expression vector, pCD-AS-hpt. The structure of pCD-AS-hpt is shown in Fig. 2. The recombinant vector pCD-AS-hpt was confirmed using HindIII/SacI and HindIII digestion, and the plasmid was sequenced to determine that no mutations in the target gene were present.

\section{Transformation of binary $\beta A S$ plant expression vector into rice}

Inflorescences of rice 'Taijing 9' at 10-15 days after pollination were collected based on the method of Chen et al. [20] to induce embryogenic callus and subculture callus once every 15 days with fresh medium.

Using the freeze-thaw method [21], the binary expression vector $\mathrm{pCD}$-AS-hpt was transformed into A. tumefaciens LBA4404, which was used to transform embryogenic calli. Co-culture of callus with Agrobacterium, screening of resistant calli and induction of differentiation of cultured calli, rooting of resistant seedlings, and other tests were conducted as described by Burkhardt et al. [22]. The medium used for Agrobacterium infection was AAM medium (pH 5.2). NB medium ( $\mathrm{pH}$ 5.8) was used for genetic transformation of rice. To screen for resistant calli, a culture medium supplemented with $50 \mathrm{mg} / \mathrm{L}$ hygromycin B (Hyg) was used [22].

\section{Identification of transgenic rice by PCR}

Genomic DNA was extracted from leaves of resistant seedlings of transgenic rice. Premix Taq ${ }^{\circ}$ Version 2.0 (Takara, D334A) was used to detect the $\beta A S$ and 


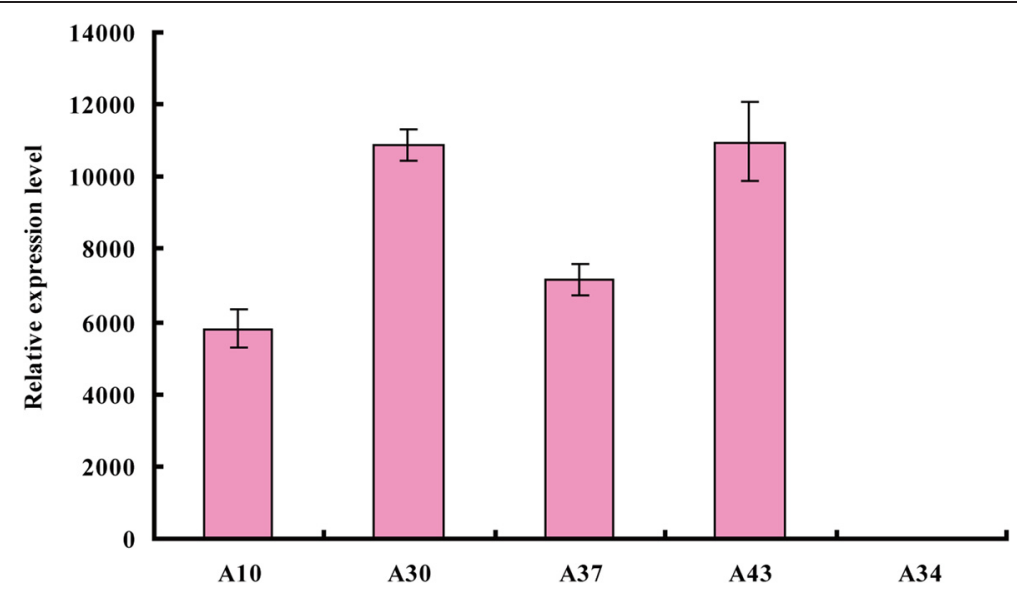

Fig. 5 Real-time fluorescence quantitative PCR analysis of $\beta A S$ expression in transgenic rice plants

hygromycin resistance $(H P T)$ genes in the transgenic rice plants. The primers used in the present study were as follows: ASF5 (5'-ATGCTTGCTTGTTGGGTTGAGG-3'), ASR2 (5' -GCCTGAATTGCTGATGAAG TGC-3'), Hpt-1 (5'-TACACAGCCATCGGTCCAGA$\left.3^{\prime}\right)$, and Hpt-2 (5'-TAGGAGGGCGTGGATATGT C$\left.3{ }^{\prime}\right)$. The PCR reaction system included a $25-\mu \mathrm{L}$ solution containing $12.5 \mu \mathrm{L}$ Premix Taq Version 2.0 (+dye), $0.5 \mu \mathrm{L}$ of each of the forward and reverse primers $(20 \mu \mathrm{M}), 2.0 \mu \mathrm{L}$ of DNA template, and $9.5 \mu \mathrm{L}$ of $\mathrm{ddH}_{2} \mathrm{O}$. The PCR reaction conditions were as follows: $94{ }^{\circ} \mathrm{C}$ for $5 \mathrm{~min} ; 94{ }^{\circ} \mathrm{C}$ for $30 \mathrm{~s}, 58{ }^{\circ} \mathrm{C}$ for $30 \mathrm{~s}$, and $72{ }^{\circ} \mathrm{C}$ for $60 \mathrm{~s} /$ $\mathrm{kb}$ for 35 cycles; and $72^{\circ} \mathrm{C}$ for $10 \mathrm{~min}$.

\section{Analysis of T-DNA insertion sites by hiTAIL-PCR}

High-efficiency thermal asymmetric interlaced PCR (hiTAIL-PCR) is a convenient way of locating T-DNA insertion sites [23]. Leaf genomic DNA was extracted from rice homozygous lines of the $\mathrm{T}_{2}$ generation. Based on the methods described by Liu et al. [22], hiTAILPCR was conducted by using Premix Ex Taq $q^{\circ}$ Version 2.0 (Takara, D335A) to amplify genomic sequences flanking the T-DNA insertion sites in transgenic rice. The clear bands that had good specificity and were > $200 \mathrm{bp}$ in size for the third PCR product were recovered for sequencing. PCR verification of the T-DNA flanking sequences was conducted by using $2 \times$ Eco Taq PCR SuperMix (TransGen Biotech, AS151). The forward primer was RB-3b (5'-GATCGCCCTTCCC AACAGT TGC-3'), and the reverse primers were A30R ( $5^{\prime}$-CAACACCCACATCGCCATCTG C-3'), A37R (5' -GCCGAGACCATCGATTCCAATG-3'), A43R1 (5'-GGCGT ATGTCTCATT GGAGGACTGC-3'), and A43R2 (5'CCAAGTACAGCCAACACTGCTGGTC-3'). The PCR reaction system included a $50-\mu \mathrm{L}$ solution containing $25.0 \mu \mathrm{L}$ of $2 \times$ Eco Taq PCR SuperMix (+dye), $1.0 \mu \mathrm{L}$ of each of the forward and reverse primers $(20 \mu \mathrm{M}), 2.0 \mu \mathrm{L}$ of the DNA template, and $21.0 \mu \mathrm{L}$ of $\mathrm{dd}_{2} \mathrm{O}$. PCR reaction conditions were $94{ }^{\circ} \mathrm{C}$ for $5 \mathrm{~min} ; 94{ }^{\circ} \mathrm{C}$ for $30 \mathrm{~s}, 58^{\circ} \mathrm{C}$ for $30 \mathrm{~s}$, and $72{ }^{\circ} \mathrm{C}$ for $60 \mathrm{~s} / \mathrm{kb}$ for 35 cycles; and $72{ }^{\circ} \mathrm{C}$ for $10 \mathrm{~min}$. The PCR products were recovered for sequencing. The resulting DNA sequences were then aligned to the rice 'Nipponbare' genome sequence (http://rice.plantbiology.msu.edu) by using the BLASTN program to determine the insertion sites and copy number of T-DNA in transgenic rice.

\section{Determination of $\beta A S$ expression levels using real-time PCR}

Total RNA was isolated from the leaves of homozygous rice lines of the $\mathrm{T}_{2}$ generation by using TRIzol ${ }^{\mathrm{Tm}}$ (Invitrogen, 15596026). Residual DNA was removed from the RNA samples by DNase I treatment (Takara, D2215). The reaction of DNase I treatment was as follows: 20$50 \mu \mathrm{g}$ of total RNA, $5 \mu \mathrm{L}$ of $10 \times$ DNase I Buffer, $2 \mu \mathrm{L}$ (10 units) of DNase I (RNase-free), 20 units of an RNase inhibitor, and DEPC-treated water to make up a total volume of $50 \mu \mathrm{L}$. DNase I treatment was conducted at $37{ }^{\circ} \mathrm{C}$ for $30 \mathrm{~min}$, and the subsequent steps were conducted as recommended by the manufacturer. A PrimeScript $^{\oplus}$ RT Reagent Kit (Takara, DRR037A) was used to reverse-transcribe total RNA to synthesize the firststrand cDNA. The conditions for reverse transcription reaction were as follows: $37{ }^{\circ} \mathrm{C}$ for $15 \mathrm{~min}$ and $85{ }^{\circ} \mathrm{C}$ for 5 s. SYBR ${ }^{\circ}$ Premix Ex Taq ${ }^{\text {Tu }}$ II (Takara, RR041A) and the ABI 7500 real-time PCR System were used for $\beta A S$ realtime PCR. Primers were qASF (5'-TGCCAGAGCAAGAAAATGGA-3'), qASR (5' -CATAGGAAGGAAAGGAGGAAGGA-3'). ACTIN served as the reference gene, and the primers were qACTF $\left(5^{\prime}\right.$-CATCTTGGCATCTCTCAGCAC-3') and qACTR (5'-AACTTTGT CCACGCTAATGAA-3'). The PCR reaction system was a $25-\mu \mathrm{L}$ solution containing $12.5 \mu \mathrm{L}$ of $2 \times \mathrm{SYBR}^{\circ}$ Premix $E x T^{T a} q^{\text {TI }}$ II, $0.5 \mu \mathrm{L}$ of each of the forward and reverse 


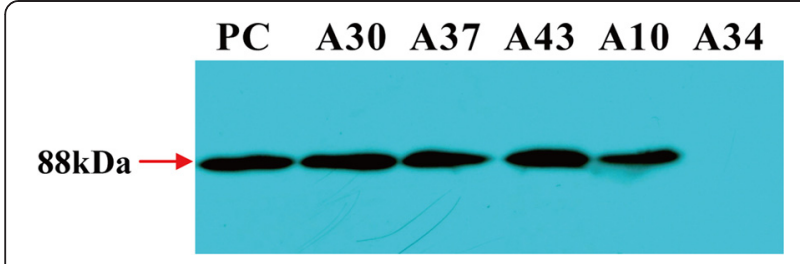

Fig. 6 Detection of the $\beta A S$ protein (about $88 \mathrm{kDa}$ ) in transgenic rice by western blotting. PC, positive control (the products of prokaryotic expression of $\beta A S)$. A34, negative rice control

primers $(20 \mu \mathrm{M}), 0.5 \mu \mathrm{L}$ of $50 \times$ ROX Reference Dye II, $5.0 \mu \mathrm{L}$ of first strand cDNA (diluted 5 times), and $6.0 \mu \mathrm{L}$ of $\mathrm{ddH}_{2} \mathrm{O}$. The PCR reaction conditions were as follows: $95{ }^{\circ} \mathrm{C}$ for $30 \mathrm{~s}$; and $95{ }^{\circ} \mathrm{C}$ for $5 \mathrm{~s}$ and $60{ }^{\circ} \mathrm{C}$ for $34 \mathrm{~s}$ for 40 cycles. The relative expression of the $\beta A S$ gene was $\mathrm{RQ}=2^{-\Delta \Delta \mathrm{Ct}}$. Each sample was amplified in triplicate.

\section{Western blot analysis of the $\beta$ AS protein}

Total protein was extracted from seeds of the $\mathrm{T}_{2}$ generation homozygous rice lines using RIPA lysis buffer (Beijing DingGuo Changsheng Biotechnology Company, Ltd., China), and total protein concentration was determined by using a total protein assay kit (Biuret Method, Shanghai Rongsheng Biotechnology Company, China). A 20-40 $\mu \mathrm{g}$ aliquot of the total protein solution was subjected to SDS-PAGE analysis by using a Mini-PRO TEAN 3 Cell (BioRad). The Proteins were then transferred to a PVDF membrane by using a Trans-Blot SD semi-dry electrophoretic transfer cell (BioRad) and transmembrane buffer ( $48 \mathrm{mmol} / \mathrm{L}$ Tris base, $39 \mathrm{mmol} / \mathrm{L}$ glycine, $0.037 \%$ sodium dodecyl sulfate (SDS), and 20\% methanol). The PVDF membrane was transferred to the TBST buffer $(10 \mathrm{mmol} / \mathrm{L}$ Tris- $\mathrm{HCl}, 100 \mathrm{mmol} / \mathrm{L} \mathrm{NaCl}$, and 0.2\% Tween-20, pH7.4) containing $5 \%$ non-fat milk and incubated at $4{ }^{\circ} \mathrm{C}$ overnight. After incubation, the PVDF membrane was reacted with the primary antibody (antiserum of the rabbit immunized with the $\beta A S$ gene product expressed in E. coli) diluted in TBST buffer containing 5\% non-fat milk (v/v 1:500) and incubated at $4{ }^{\circ} \mathrm{C}$ overnight. The PVDF membrane was then washed with TBST buffer. The secondary antibody (HRP-labeled goat anti-rabbit IgG, Beijing DingGuo Changsheng Biotechnology Company, Ltd., China) was diluted with TBST buffer (v/v 1:5,000), and the PVDF membrane was transferred to the above solution and incubated at room temperature for $1 \mathrm{~h}$. The PVDF membrane was then washed with TBST buffer. The hybridization signal was developed by using the SuperSensitive ECLsolution (Pierce) and exposed for $1 \mathrm{~min}$ by using an X-ray film (Kodak) in a dark room [19]. Rabbit polyclonal antibody against $\beta$ AS (primary antibody) was prepared by GL Biochem Co., Ltd. (Shanghai, China).
Analysis of sapogenin content in transgenic rice by HPLC The rice grains from the $T_{2}$ generation homozygous rice lines were ground to powder and passed through an 80mesh screen. Approximately $0.2 \mathrm{~g}$ of each sample was then transferred into a round flask to which $4 \mathrm{~mL}$ of methanol was added. The round flask was connected to a Dimroth's condensing tube and reflux extraction was conducted in an $80{ }^{\circ} \mathrm{C}$ water bath for $12 \mathrm{~h}$. Methanol was added to the extraction solution to a final volume of $5 \mathrm{~mL}$. The solution was filtered through a $0.45-\mu \mathrm{m}$ organic filter membrane and then subjected to HPLC analysis $[24,25]$.

The LC-20A high-performance liquid chromatography, SPD-M20A UV-vis detector and the software LC solution 1.24 SP1 (Shimadzu, Japan) were used to determine the concentration of sapogenin using oleanolic acid (Ole) as the standard. The column was a Hypersil ODS2 C18 column $(250 \mathrm{~nm} \times 4.6 \mathrm{~nm}, 5 \mu \mathrm{m}$; Dalian Elite Company, China), the chromatographic conditions were as follows: mobile phase was methanol $-0.05 \mathrm{~mol} / \mathrm{L}$ $\mathrm{NaH}_{2} \mathrm{PO}_{4}$ (85:15), flow rate was $1.0 \mathrm{ml} / \mathrm{min}$, column temperature was $30{ }^{\circ} \mathrm{C}$, and the detection wavelength was $210 \mathrm{~nm}$ [24-26]. Standard oleanolic acid (Batch No.: 110709-200505) was purchased from the Chinese Academy of Food and Drug Testing.

\section{Results and discussion}

\section{Generation of transgenic rice}

The digestion products of the binary plant expression vector pCD-AS-hpt were of the expected sizes. Sequencing of pCD-AS-hpt showed no mutations in the $\beta A S$ gene. These findings were indicative of the successful construction of the binary plant expression vector $\mathrm{pCD}$ AS-hpt.

The binary vector was transferred to rice 'Taijing 9', and 302 anti-Hyg rice plants of the $\mathrm{T}_{0}$ generation were generated. The PCR results of $H P T$ and $\beta A S$ (Fig. 3) indicated that all resistant seedlings contained the $H P T$ gene, whereas 68 plants contained $\beta A S$ (positive rate: $22.5 \%)$. No significant differences in agronomic traits or appearance between the offspring of transgenic rice and the receiver 'Taijing 9' were observed.

\section{Confirmation of T-DNA insertion sites by hiTAIL-PCR}

hiTAIL-PCR analysis showed clear bands with good specificity in the third round of PCR products from three lines of the $T_{2}$ generation homozygous rice lines, whereas that of the other lines were vague or smaller than 200 bp. Sequencing (GenBank Acc. Nos.: KP687751, KP687752, KP687753, and KP687754), PCR verification (Fig. 4), and alignment with the rice 'Nipponbare' genome sequence showed that the transgenic rice lines A30 and A37 both had one copy of the $\beta A S$ gene-harboring 

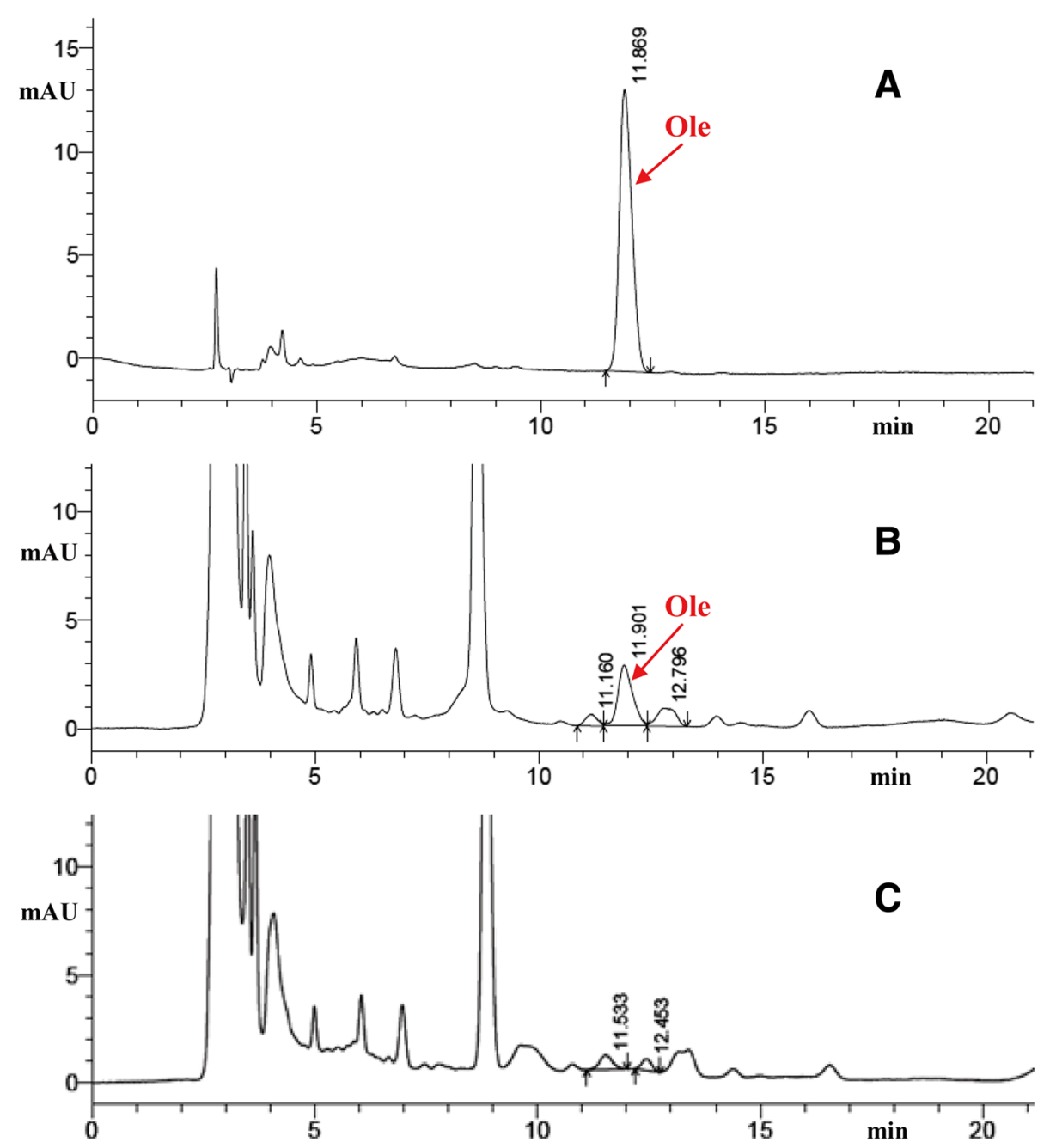

Fig. 7 HPLC chromatograms of oleanolic acid. a Ole standard. b HPLC chromatograms of Ole in transgenic rice plants. c non-transgenic control rice

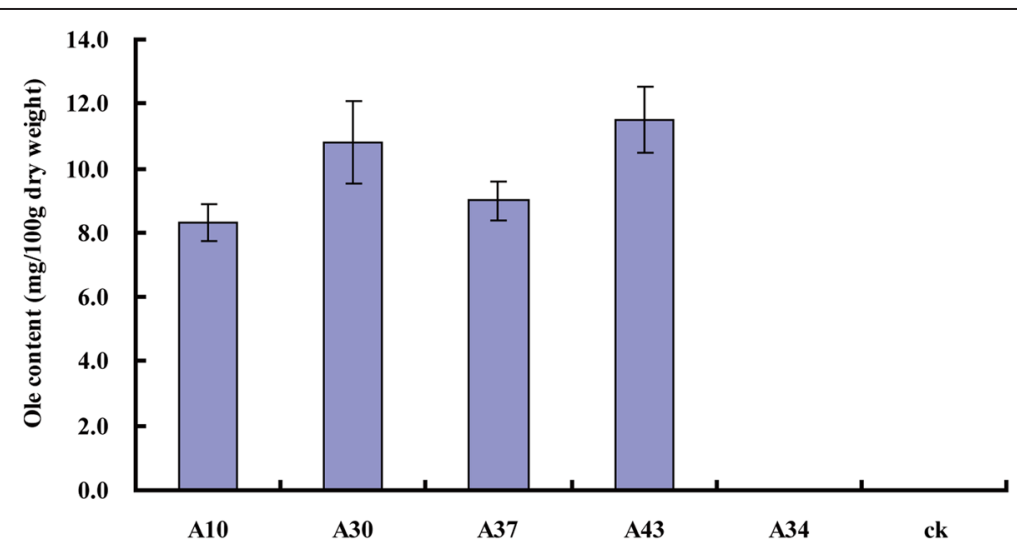

Fig. 8 Oleanolic acid production as analyzed by HPLC. (ck), non-transgenic control rice 
T-DNA, whereas line A43 had two copies of the transgene. The insertion sites in these three lines were located at 9,533,306 bp in chromosome 5, 5,429,409 bp in chromosome 12, and 32,736,409 bp and 32,740,184 bp in chromosome 2 , respectively.

Previous studies have shown that transgenic plants harbor a few copies of T-DNA insertions, with an average of 1.2-2.0 copies, and most have only a single copy $[27,28]$. In the present study, various homozygous lines of the $\mathrm{T}_{2}$ generation harbored 1-2 copies of T-DNA, and the insertion sites of T-DNAs varied. This also confirms that T-DNA insertion is a relatively random event $[29,30]$.

\section{Overexpression of exogenous $\beta A S$ in transgenic rice}

Real-time PCR analysis showed that the $\beta A S$ gene was overexpressed in the $T_{2}$ generation homozygous lines A10, A30, A37, and A43. The relative expression level was within the range of 5782.1-10957.8 (Fig. 5, A34 was a negative line).

\section{Western blot analysis of $\beta$ AS protein}

The rice positive samples of the $T_{2}$ generation homozygous lines A10, A30, A37, and A43 and the positive control (prokaryotic expression product of $\beta A S$ ) showed the expected hybridization band of about $88 \mathrm{kDa}$ in size. This specific band was not detected in the negative sample A34 line (Fig. 6). These results indicated that the seeds of the transgenic rice expressed $\beta$ AS.

Analysis of sapogenin content in transgenic rice by HPLC HPLC analysis indicated that the seeds of the $\mathrm{T}_{2}$ generation homozygous lines A10, A30, A37, and A43 had an Ole content ranging from 8.3 to $11.5 \mathrm{mg} / 100 \mathrm{~g} \mathrm{dw}$ (Figs. 7 and 8).

\section{Transgenic yeast and rice as new sources of ginsenosides} Because supplies of wild ginseng have been depleted, ginsenosides are mainly extracted from cultivated ginseng. However, artificial cultivation is relatively timeconsuming and does not allow continuous cropping, preventing ginsenoside production from meeting the increased demand of the community. Therefore, it is necessary to explore new sources of ginsenosides for medicinal use $[8,9]$. Scientists utilize synthetic biology to synthesize ginsenosides. Cloning and characterization of a key enzyme in the synthesis of 20 (S)-protopanaxadiol (PPD) has resulted in the construction of a yeast cell capable of producing PPD [31]. Cytochrome P450 was found to catalyze PPD to produce $20(\mathrm{~S})$-protopanaxatriol (PPT) [32]. A "ginseng yeast" capable of simultaneously producing three kinds of ginsenosides, Ole, PPD, and PPT has also been constructed, with yields of $21.4 \mathrm{mg} / \mathrm{L}$, $17.2 \mathrm{mg} / \mathrm{L}$, and $15.9 \mathrm{mg} / \mathrm{L}$, respectively [33, 34]. The rare ginsenoside, compound $\mathrm{K}(\mathrm{CK})$, which has not been detected in ginsenosides, is the active metabolite that has been detected in the blood after oral administration of ginseng and other ginsenosides [35]. Yan et al. previously reported that ginseng UDP-glycosyltransferase (PgUGT1) could specifically catalyze glycosylation of the C-20S hydroxyl group in dammarane-type tetracyclic triterpenoids, and yeast cells synthesized CK from monosaccharides when PgUGT1 was co-expressed in the PPD synthesis pathway [36].

Adding the biosynthetic branch of new metabolites to rice by using genetic engineering facilitates the synthesis of new active ingredients. Modifying existing metabolic pathways in rice to increase the concentration of specific components to improve its nutritional value has become an important means of creating new germplasm. A number of important advances such as "golden rice" $[5,6]$, "high-iron rice" [37, 38], and "sesame nutrition rice" [7] can be attributed to this technique. In the present study, a new rice germplasm that produces oleanane-type sapogenin was produced by expressing the $P$. japonicus $\beta A S$ gene. This germplasm can serve as a new resource for breeding "ginseng rice" varieties, as well as create new ginseng saponin donors. Because rice is a food crop that is simpler to cultivate than the ginseng genus plants, it is generally easier to produce saponins in modified strains, thus allowing more people to benefit from this novel production approach. Improvement in the standard of living has resulted higher production requirements for medicine and food. The saponins synthesized by plants might be safer and more efficient than those produced via cellular engineering. Therefore, the results of the present study will have a major impact on the genetic breeding of functional rice, as well as on research and development of sources of medical ginsenosides.

\section{Conclusions}

This is the first report on the transformation of the $P$. japonicus $\beta A S$ gene into rice and the generation of a new "ginseng rice" germplasm that produces oleananetype sapogenin. The concentration of Ole in transgenic rice was within the range of $8.3-11.5 \mathrm{mg} / 100 \mathrm{~g} \mathrm{dw}$. The current results indicate that it is feasible to breed new varieties of "ginseng rice" and create new ginsenoside donors by transforming key genes in the ginsenoside biosynthesis pathway.

\section{Abbreviations}

BAS: $\beta$-Amyrin synthase; T-DNA: Transfer-DNA; P. japonicus: Panax japonicus; P. ginseng: Panax ginseng; P. quinquefolius: Panax quinquefolius; FPP: Farnesyl pyrophosphate; SQS: Squalene synthase; SQE: Squalene epoxidase; CS: Cycloartenol synthase; LS: Lanosterol synthase; aAS: a-Amyrin synthase; LUS: Lupeol synthase; DS: Dammarenediol-II synthase; OSCs: 2,3-Oxidosqualene cyclases; 355 polyA: Terminator of CaMV 355 gene; HPT: Hygromycin phosphotransferase gene; $35 \mathrm{~S} \mathrm{P:} 35 \mathrm{~S}$ promoter; 
NOS T: Terminator of the nopaline synthase gene; Ubi P: Ubiquitin promoter; LB: Left border; RB: Right border; E. coli: Escherichia coli; A. tumefaciens: Agrobacterium tumefaciens; Hyg: Hygromycin B; hiTAlL-PCR: High-efficiency thermal asymmetric interlaced PCR; HPLC: High-performance liquid chromatography; SDS-PAGE: Sodium dodecyl sulfate polyacrylamide gel electrophoresis; PC: Positive control; Ole: Oleanolic acid; PPD: 20 (S)-Protopanaxadiol; PPT: 20 (S)-Protopanaxatriol; CK: Compound K; PgUGT1: Panax ginseng UDP-glycosyltransferase.

\section{Competing interests}

The authors declare that they have no competing interests.

\section{Authors' contributions}

JZ designed the study; ZH performed the experiment, conducted the analysis, and wrote the paper; $J \mathrm{~L}$ helped perform the experiments and analyze data; $\mathrm{ZC}, \mathrm{MX}, \mathrm{MG}, \mathrm{XH}$, and $\mathrm{ZY}$ helped perform the experiments. All authors have read and approved the final manuscript.

\section{Acknowledgements}

The National Transgenic Organism New Variety Culture Key Project (2009ZX08001-032B), the National Key Technology R\&D Program (2013BAD01B05), and the Major Science and Technology Projects of Fujian Province (2013NZO002-2) in China financially supported this research study.

\section{Author details}

${ }^{1}$ College of Food Science, Fujian Agriculture and Forestry University, 15 Shangxiadian Road, CangShan District, Fuzhou 350002, Fujian, China. ${ }^{2}$ Agricultural Product Quality Institute, Fujian Agriculture and Forestry University, 15 Shangxiadian Road, CangShan District, Fuzhou 350002, Fujian, China.

\section{Received: 6 February 2015 Accepted: 15 May 2015}

\section{Published online: 02 June 2015}

\section{References}

1. Khush GS. What it will take to Feed 5.0 Billion Rice consumers in 2030. Plant Mol Biol. 2005;59(1):1-6.

2. Peng S, Laza RC, Visperas RM, Sanico AL, Cassman KG, Khush GS. Grain yield of rice culitvars and lines developed in Philippines since 1996. Crop Sci. 2000;40(2):307-14.

3. Sofi P, Rather AG, Zargar MY. Breaking the yield barrier of rice: remodeling photosynthesis. Am J Plant Physiol. 2006;1 (2):113-26.

4. Christou P, Ford TL, Kofron M. Production of transgenic rice (Oryza Sativa L.) plants from agronomically important Indica and Japonica varieties via electric discharge particle acceleration of exogenous DNA into immature zygotic embryos. Bio/Technology. 1991;9(10):957-62.

5. Ye XD, Al-Babili S, Klöti A, Zhang J, Lucca P, Beye P, et al. Engineering the provitamin A (beta-carotene) biosynthetic pathway into (carotenoid-free) rice endosperm. Science. 2000;287(5451):303-5.

6. Paine JA, Shipton CA, Chaggar S, Howells RM, Kennedy MJ, Vernon G, et al. Improving the nutritional value of Golden Rice through increased pro-vitamin A content. Nat Biotechnol. 2005;23(4):482-7.

7. Lee TTT, Chung M, Kao Y, Wang C, Chen L, Tzen JTC. Specific expression of a sesame storage protein in transgenic rice bran. J Cereal Sci. 2005;41(1):23-9.

8. Li FY. Chinese Ginseng and American Ginseng. Beijing: China Agricultural Science and Technology Press; 2002 (in Chinese).

9. Yun TK. Panax ginseng-a non-organ-specific cancer preventive? Lancet Oncol. 2001;2(1):49-55

10. Haralampidis K, Trojanowska M, Osbourn AE. Biosynthesis of triterpenoid saponins in plants. Adv Biochem Eng Biotechnol. 2002;75:31-49.

11. Hao HL, Zhu XF, Zeng YZ. The biosynthesis and regulation of isoprenoids. J Zhejiang Agric Univ (Agric Life Sci). 2002;28(02):224-30 (in Chinese).

12. Liang YL, Zhao SJ. Progress in understanding of ginsenoside biosynthesis. Plant Biol. 2008;10(4):415-21.

13. Chappell J. Biochemistry and molecular biology of the isoprenoid biosynthetic pathway in plants. Annu Rev Plant Physiol Plant Mol Biol. 1995;46:521-47.

14. Chen DH, Ye HC, Li GF, Liu Y. Advances in molecular biology of plant isoprenoid metabolic pathway. Acta Bot Sin. 2000;42(06):551-8 (in Chinese).
15. Zhao SJ, Hou CX, Xu LX, Liang YL, Qian YC, Yao S. Effects of suppressing oleanane-type ginsenoside biosynthesis on dammarane-type ginsenoside production. J Jilin Univ (Eng Tech Ed). 2011;41(3):865-8.

16. Suzuki H, Achnine L, Xu R, Matsuda SPT, Dixon RA. A genomics approach to the early stages of triterpene saponin biosynthesis in Medicago truncatula. Plant J. 2002;32(6):1033-48.

17. Haralampidis K, Bryan G, Qi X, Papadopoulou K, Bakht S, Melton R, et al. A new class of oxidosqualene cyclases directs synthesis of antimicrobial phytoprotectants in monocots. Proc Natl Acad Sci U S A. 2001;98(23):13431-6.

18. Augustin JM, Kuzina V, Andersen SB, Bak S. Molecular activities, biosynthesis and evolution of triterpenoid saponins. Phytochemistry. 2011;72(6):435-57.

19. Sambrook J, Russell DW, translated by Huang PT. Molecular Cloning: A Laboratory Manual. 3rd ed. Beijing: Science Press; 2002 (in Chinese).

20. Chen L, Zhang S, Beachy RN, Fauquet CM. A protocol for consistent, largescale production of fertile transgenic rice plants. Plant Cell Rep. 1998;18:25-31

21. Hofgen R, Willmitzer L. Storage of competent cells for Agrobacterium transformation. Nucleic Acids Res. 1988;16(20):9877.

22. Burkhardt PK, Beyer P, Wunn J, Kloti A, Armstrong GA, Schledz M, et al. Transgenic rice (Oryza sativa) endosperm expressing daffodil (Narcissus pseudonarcissus) phytoene synthase accumulates phytoene, a key intermediate of provitamin A biosynthesis. Plant J. 1997;11(5):1071-8.

23. Liu YG, Chen YL. High-efficiency thermal asymmetric interlaced PCR for amplification of unknown flanking sequences. Biotechniques. 2007:43(5):649

24. Liu W, Ding HJ. Determination of Ursolic Acid, Oleanolic Acid and Rosmarinic Acid in Prunella vulgaris L. by HPLC. Chinese Traditional Patent Medicine. 2008;30(04):577-80

25. Wei JH, Li YH, Cai SF, Yang XM, Zhen HS. Determination of Oleanolic Acid and Ursolic Acid in Diospyros kaki by HPLC. Chinese Journal of Experimental Traditional Medical Formulae. 2010;16(13):48-50.

26. Wang HH, Wang ZZ, Guo WB. Comparative determination of ursolic acid and oleanolic acid of Macrocarpium officinalis (Sieb. et Zucc.) Nakai by RP-HPLC. Ind Crop Prod. 2008;28(3):328-32.

27. Jeon JS, Lee $\mathrm{S}$, Jung $\mathrm{KH}$, Jun $\mathrm{SH}$, Jeong $\mathrm{DH}$, Lee J, et al. T-DNA insertional mutagenesis for functional genomics in rice. Plant J. 2000;22(6):561-70.

28. Krishnan A, Guiderdoni E, An G, Hsing YC, Han CD, Lee MC, et al. Mutant resources in rice for functional genomics of the grasses. Plant Physiol. 2009;149(1):165-70.

29. Azpiroz-Leehan R, Feldmann KA. T-DNA insertion mutagenesis in Arabidopsis: going back and forth. Trends Genet. 1997;13(4):152-6.

30. Krysan PJ, Young JC, Sussman MR. T-DNA as an insertional mutagen in Arabidopsis. Plant Cell. 1999;11(12):2283-90.

31. Han JY, Kim HJ, Kwon YS, Choi YE. The Cyt P450 enzyme CYP716A47 catalyzes the formation of protopanaxadiol from dammarenediol-II during ginsenoside biosynthesis in Panax ginseng. Plant Cell Physiol. 2011;52(12):2062-73.

32. Han JY, Hwang HS, Choi SW, Kim HJ, Choi YE. Cytochrome P450 CYP716A53v2 catalyzes the formation of protopanaxatriol from protopanaxadiol during ginsenoside biosynthesis in Panax Ginseng. Plant Cell Physiol. 2012;53(9):1535-45.

33. Dai ZB, Liu Y, Zhang XN, Shi MY, Wang BB, Wang D, et al. Metabolic engineering of Saccharomyces cerevisiae for production of ginsenosides. Metab Eng. 2013;20:146-56.

34. Dai ZB, Wang BB, Liu Y, Shi MY, Wang D, Zhang XN, et al. Producing aglycons of ginsenosides in bakers' yeast. Sci Rep. 2014;4:3698.

35. Hasegawa H. Proof of the mysterious efficacy of ginseng: basic and clinical trials: metabolic activation of ginsenoside: deglycosylation by intestinal bacteria and esterification with fatty acid. J Pharmacol Sci. 2004;95(2):153-7.

36. Yan X, Fan Y, Wei W, Wang PP, Liu QF, Wei YJ, et al. Production of bioactive ginsenoside compound $\mathrm{K}$ in metabolically engineered yeast. Cell Res. 2014;24(6):770-3.

37. Goto F, Yoshihara T, Shigemoto N, Toki S, Takaiwa F. Iron fortification of rice seed by the soybean ferritin gene. Nat Biotechnol. 1999;17(3):282-6.

38. Lucca P, Hurrell R, Potrykus I. Genetic engineering approaches to improve the bioavailability and the level of iron in rice grains. Theor Appl Genet. 2001;102(2-3):392-7. 